\title{
Periprosthetic Joint Infection
}

\section{Editorial Comment}

\author{
Javad Parvizi MD, Craig J. Della Valle MD
}

Published online: 3 December 2010

(C) The Association of Bone and Joint Surgeons (B) 2010

Total joint arthroplasty has indeed come a long way. From the days when this surgery was only for elderly and mostly sedentary individuals, it is now offered to all ages and at times to extremely active patients. The widespread use of joint replacement is mostly due to its unparalleled success. The collaboration between surgeons and industry has allowed us to largely address many of the issues that haunted joint replacement in the early era such as extensive bearing surface wear and osteolysis. With the exception of occasional higher rates of complications with a few specific devices, we have continued to make progress and clear many milestones.

One area that continues to challenge arthroplasty surgeons and places an immense burden on patients and society is periprosthetic joint infection (PJI). Although the incidence of this dreaded complication is low (1-2\% in North America-based on registry data), the financial and psychological costs are high. There is emerging evidence that PJI is on the rise and the success for treatment of this condition has continued to decline. There may be a multitude of reasons for the latter, the most important of which likely relates to a rise in the incidence of PJI caused by resistant organisms. In addition, recent research demonstrates some failures thought to have been aseptic may indeed be failures secondary to PJI that have escaped diagnosis using available diagnostic modalities.

\section{J. Parvizi ( $ه)$}

The Rothman Institute, 925 Chestnut Street,

Philadelphia, PA 19107, USA

e-mail: parvj@aol.com

\section{J. Della Valle}

Department of Orthopaedic Surgery, Rush University

Medical Center, Chicago, IL, USA

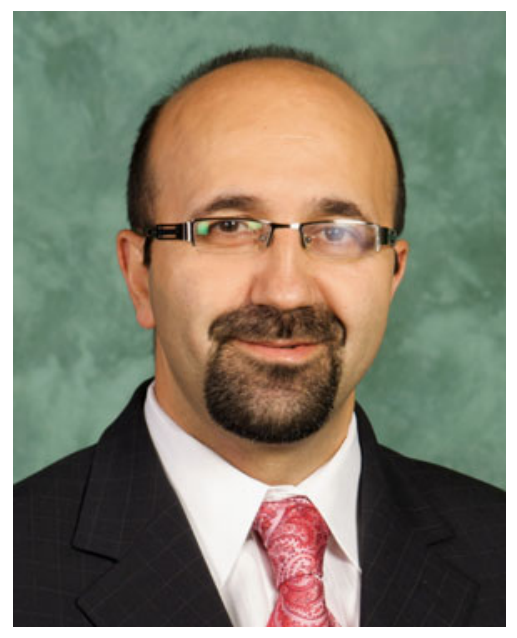

Fig. 1 Javad Parvizi, MD, is shown.

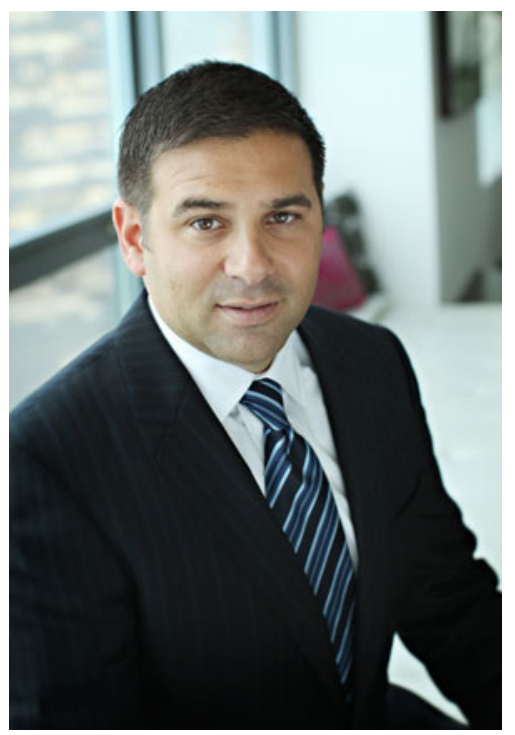

Fig. 2 Craig J. Della Valle, MD, is shown. 
The coming decade holds a great challenge for orthopaedic surgeons in general, and those performing joint arthroplasty in particular. The greatest challenges are to prevent this dreaded complication and provide surgical treatment with a higher rate of success. Another challenge is to design techniques that allow us to diagnose PJI quickly and with a higher degree of certainty.

In this Symposium we have assembled an accomplished group of experts to present their scientific findings pertinent to all of these challenges. These internationally recognized and respected experts have provided valuable information to the readers and some have enumerated the current difficulties that face us. Let us hope that we will all be able to face the challenges of periprosthetic joint infection that threaten our patients and the success of a surgical procedure that alleviates pain and restores function to millions of patients every year. 